\title{
Latex immunotherapy: evidence of effectiveness
}

\author{
Eleonora Nucera ${ }^{1}$, Simona Mezzacappa ${ }^{1}$, Alessandro Buonomo ${ }^{2}$, Michele Centrone ${ }^{2}$, Angela Rizzi ${ }^{2}$, \\ Paolo Francesco Manicone ${ }^{3}$, Giampiero Patriarca ${ }^{1}$, Arianna Aruannoํ․ Domenico Schiavino ${ }^{1}$
} ${ }^{1}$ Allergy Unit, Università Cattolica del Sacro Cuore - Fondazione Policlinico “A. Gemelli”, Rome, Italy
2Department of Head and Neck, Division of Oral Surgery and Implantology, Fondazione Policlinico Universitario Gemelli, Rome, Italy
3Department of Oral Sciences, Catholic University, Rome, Italy

Adv Dermatol Allergol 2018; XXXV (2): 145-150

DOI: https://doi.org/10.5114/ada.2018.75235

\begin{abstract}
Introduction: The only etiological and decisive therapy, able to influence the natural history of latex allergy is the specific desensitization.

Aim: To verify the clinical efficacy and immunological changes determined by latex sublingual immunotherapy in allergic patients who underwent this treatment for at least 3 years.

Material and methods: We enrolled 76 patients (16 males and 60 females, mean age 34 years old) with evidence of a natural rubber latex allergy. To assess the effectiveness of the immunotherapy we performed a latex skin prick test, specific IgE and IgG4 and challenge tests before and after at least 3 years of desensitization.

Results: We observed a reduction in the mean diameter of the wheal area at the skin prick test and a decrease in latex specific IgE while no significant changes of latex IgG4 values were found. Moreover a reduction of symptoms and scores at the provocation tests were remarked.

Conclusions: Although the primary prevention (which still remains the gold standard treatment for patients suffering from the latex allergy) sublingual immunotherapy can be offered with efficacy in addition to symptomatic treatment to selected patients.
\end{abstract}

Key words: latex allergy, sublingual immunotherapy, challenge, desensitization.

\section{Introduction}

Allergic reactions to natural rubber latex (NRL) have been reported since 1970 and during the last decades they became an emerging clinical problem of public health. Symptoms of a NRL allergy range from contact urticaria and asthma to anaphylaxis and they are elicited by direct contact with NRL items (i.e. medical devices) or by inhalation of latex airborne proteins.

The prevalence rates of sensitization in pediatric and adult population are $0.3 \%$ and $0.7 \%$, respectively [1]. The latex allergy mostly affects certain groups of population with a high risk of sensitization (healthcare workers, patients with frequent hospitalization, etc.) and recently there has been an increasing number of cases of the NRL allergy in other occupations such as hairdressers, housekeepers, security personnel, etc. [2], probably due to widespread exposure to latex gloves.

The diagnosis of the latex allergy is formulated on the basis of personal history and on an accurate allergological evaluation; the patients with a history of adverse reactions during medical or surgical procedures, food intolerance or risk factors should undergo a latex skin prick test (SPT), latex specific IgE (slgE) assay and challenge tests (nasal, conjunctival, bronchial, intravaginal, sublingual, cutaneous).

Proper diagnosis of the latex allergy is important for appropriate preventive measures and treatment [3].

In fact, the progressive corrective measures adopted, including the substitution of NRL with other materials or the use of powder-free gloves, resulted in a decline in incidence of this disease [4].

Actually the complete avoidance of NRL is recommended to reduce the risk of symptoms onset in allergic patients; however, in some subjects this measure is not sufficient and it is complicated by the ubiquity of latex products and by the cross-reactions to latex and fruit/ vegetables (latex-fruit syndrome).

The only etiological and decisive therapy, able to influence the natural history of latex allergy is the specific desensitization. Only few studies have been published regarding its efficacy and tolerance.

Address for correspondence: Dr. Arianna Aruanno, Allergy Unit, Università Cattolica del Sacro Cuore, Policlinico “A. Gemelli”, Largo F. Vito, 1-00168 Rome, Italy, phone: +39 06 30155896, fax: +39 06 30156999, e-mail: aarianna@hotmail.it Received: 4.09.2016, accepted: 15.02.2017. 
Table 1. Rush sublingual desensitization treatmnent to latex

\begin{tabular}{lccc}
\hline Day & Concentration & Administered dose & Total dose \\
\hline 1 & From $10^{-18}$ to $10^{-10}$ & 1 drop every administration & 28 per $10^{-10} \mu \mathrm{g}$ of NRL \\
\hline 2 & From $10^{-9}$ to $10^{-1}$ & 1 drop every administration & $2.8 \mu \mathrm{g}$ of NRL \\
\hline 3 & Undiluted solution $(500 \mu \mathrm{g} / \mathrm{ml})$ & $1,2,3,4,5,10$ drops & $500 \mu \mathrm{g}$ of NRL \\
\hline
\end{tabular}

Maintenance treatment: 10 drops 3 times a week.

Our previous study [5] showed an improvement in skin and respiratory symptoms and in conjunctival challenge tests after the desensitization. At the end of the therapy, in fact, almost all patients were able to wear latex gloves, to undergo medical or surgical intervention, to stay in environments where latex is present. Our protocol of rush latex desensitization treatment (Table 1) was performed in 4 days, during which increasing doses of the latex extract (Alk-Abellò, Milan) were administered under the patient's tongue until reaching the highest dose of $500 \mu \mathrm{g}$ of latex during hospitalization. A maintenance therapy (10 drops of an undiluted solution three times a week) was followed at home. All the patients were equipped with an emergency kit which included auto injectable epinephrine, betamethasone and clorphenamine and we suggested future specialist visits in latex-safe environment before latex tolerance was acquired.

In another open-label and non-controlled study, Cistero [6], in a trial of 10 weeks, demonstrated a higher tolerability of the sublingual than subcutaneous route. In a double-blind, placebo-controlled study of 1-year sublingual immunotherapy, Nettis [7] showed a significant improvement in symptoms and medication scores after the 12 months' treatment. Finally, another trial suggested that latex sublingual immunotherapy (SLIT) was more effective than placebo after 1-year follow-up, but only 9 patients completed the study and, hence, results must be treated with caution [8].

All these studies described in literature have some deficiencies (an inappropriate trial design, short followup or the use of different commercial products). Thus, given that we decided to verify the clinical efficacy and immunological changes determined by NRL immunotherapy in allergic patients who underwent this treatment for at least 3 years, as happened in respiratory immunotherapy $[9,10]$ and food allergy [11].

\section{Material and methods}

\section{Trial design}

This study is an observational trial and its aim is to verify the clinical efficacy of NRL SLIT (Alk-abellò) in patients who finished the treatment or were treated for at least 3 years.
The primary endpoint was assessed by the changes in the response to challenge tests (cutaneous, mucousoral, conjunctival, nasal), performed before and after 3 years of therapy.

The secondary endpoint was to evaluate the possible immunological changes determined by the immunotherapy by means of skin prick tests with latex (Alk-abellò, Milan), and the assay of latex specific IgE and IgG4.

\section{Patients}

The study was carried out on all the subjects referred to the Allergy Department of Policlinico "A. Gemelli" in Rome with evidence of an NRL allergy (positive SPT and specific IgE). The diagnosis of the latex allergy was confirmed by challenge tests (cutaneous, mucous-oral, nasal, and conjunctival). All subjects were questioned about symptoms after latex exposure (ranging from contact urticaria to anaphylaxis) and possible risk factors (atopy; occupation as healthcare workers, dentists, rubber plantation workers, etc.; multiple surgical operations; myelomeningocele).

The inclusion criteria were: age 18-60 years, an immediate reaction after latex exposure, positive skin prick test for latex (Alk-abellò) (> $3 \mathrm{~mm}$ ), positive latex specific IgE assay (>0.35 KU/I) (Phadia, Uppsala, Sweden) and positive latex challenge.

The exclusion criteria were ongoing breastfeeding or pregnancy, positive placebo challenge and any clinical condition that contraindicates immunotherapy according to the European Academy of Allergy and Clinical Immunology (EAACI) guidelines [12].

Written informed consent was obtained from all patients.

Every patient signed informed consent to perform challenge tests and subsequently to undergo the desensitization treatment.

\section{Skin prick test}

All patients underwent the skin prick test (SPT) with the NRL extract (Alk-Abellò, Milan, Italy). All SPT were performed and read after 15 min according to the EAACI guidelines [13]: a wheal diameter greater than or equal to $3 \mathrm{~mm}$ was considered as a positive result. Skin prick test with a histamine $(10 \mathrm{mg} / \mathrm{ml})$ and saline solution were carried out as positive and negative controls, respectively. 


\section{In vitro parameters}

Serum samples were collected before and after the treatment for the assay of latex specific IgE and IgG4 by the UniCAP System (Thermo-fisher); samples with specific IgE concentrations of $\geq 0.35 \mathrm{KU} / \mathrm{l}$ were considered as positive.

\section{Challenge tests [14]}

Latex provocation tests were performed in a day hospital regimen under physician observation and vital parameters were monitored during each test.

The cutaneous provocation test consisted of wearing a latex glove (Triflex Allegiance Health Care Co., McGaw Park, IL, USA) on one hand for $60 \mathrm{~min}$.

The mucous-oral challenges were carried out by asking the patient to hold a latex-gloved test tube in the mouth until symptoms appeared or up to $1 \mathrm{~h}$.

The conjunctival and nasal challenges were performed by instilling latex into the inferior fornix of alternate eyes or by inhaling latex solutions of the commercial extract, starting with the concentration of $500 \times 10^{-8}$ $\mu \mathrm{g} / \mathrm{ml}$ up to $50 \mu \mathrm{g} / \mathrm{ml}$.

During latex challenge tests, ocular (tearing, itching, erythema and conjunctival edema), respiratory (rhinorrhea, nasal itching, nasal blockage, sneezing, wheezing, cough, dyspnea and laryngospasm) and muco-cutaneous symptoms (generalized pruritus, erythematous-papular rash, urticarial-angioedema, oral and pharyngeal itching) were recorded by the same physicians who observed the patient until the end of each challenge and assessed score symptoms on a four-point scale: 0 (absent), 1 (mild), 2 (moderate) and 3 (severe). Also a medication score, assessing administration of symptomatic medication during these provocation tests, was calculated accordingly to each therapeutic category: 1 point for local anti-allergic treatment, 2 points for an antihistamine tablet and 3 points for parenteral therapy (steroids or antihistamines).

\section{Immunotherapy schedule}

A rush sublingual desensitizing treatment with latex was performed in 4 days by administering increasing doses of the latex extract under the patients' tongue [8]. The patients were instructed to keep the allergen solution in the mouth for at least 3 min and then swallow it. The protocol started with a drop of the latex solution $(500 \mu \mathrm{g} / \mathrm{ml})$ diluted $1: 10^{18}$ until reaching the highest dose of $1 \mathrm{ml}$ of the undiluted solution ( $500 \mathrm{\mu g}$ of latex). A maintenance therapy $(500 \mu \mathrm{g}$ of undiluted solution three times a week) was recommended (Table 1).

All patients were equipped with a diary, where they were asked to indicate possible side effects relating to SLIT, an emergency kit (antihistamines, corticosteroids, auto injectable epinephrine) to manage adverse reac- tions and we suggested future specialist visits in latexsafe environment.

The treatment started in a Day Hospital regimen and, when the patients reached the maintenance dose, continued at home with monthly hospital visits to hand back the diary to monitor the compliance.

\section{Statistical analysis}

We performed the McNemar's $\chi^{2}$ test and the Wilcoxon-Mann-Whitney test to evaluate the results of the challenges and the changes in skin prick tests, latex specific IgE and IgG4 before and after the desensitization. The significance level was set at $p>0.05$.

\section{Results}

We enrolled 76 patients ( 16 males and 60 females, mean age 34 years old) with evidence of the NRL allergy who underwent desensitization treatment for at least 3 years.

At time of diagnosis, all but two patients were positive to the skin prick test with latex and 67 out of the 76 subjects presented positive latex specific IgE. These patients fulfilled inclusion criteria because the latex allergy diagnosis was confirmed by challenge tests.

After the desensitization treatment, we observed a negative latex SPT only in 9 patients but in 91\% of our population we observed a decrease in the mean diameter of the wheal area from $7.78 \mathrm{~mm}$ before the SLIT to $5.24 \mathrm{~mm}$ after SLIT ( $p<0.001$ ) (Figure 1).

Considering serum levels of latex specific IgE after desensitization we detected a value $<0.35 \mathrm{kU} / \mathrm{l}$ in $10 \mathrm{pa}$ tients although a reduction in this value was measured in $78 \%$ of patients (the mean value of specific IgE was $13.33 \mathrm{KU} / \mathrm{I}$ before the SLIT and $8.95 \mathrm{kU} / \mathrm{l}$ after the SLIT, $p<0.01$ ) (Figure 2).

The values of specific lgG4 did not show significant changes after desensitization.

The results of challenges are presented in Table 2.

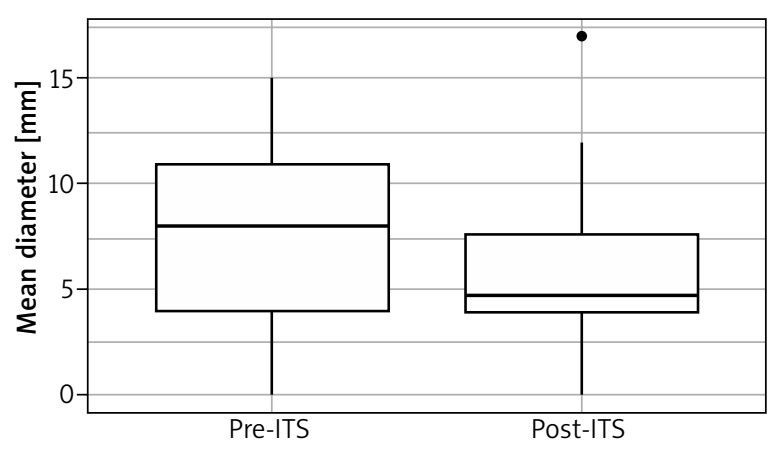

Figure 1. Latex SPT before and after sublingual immunotherapy (ITS) 
Table 2. Results of challenge tests

\begin{tabular}{lcc}
\hline Variable & Positive before immunotherapy & Positive after immunotherapy \\
\hline Latex skin prick test $(n=76)$ & 74 & 65 \\
\hline Latex slgE $(n=76)$ & 67 & 57 \\
\hline Cutaneous challenge test $(n=72)$ & 57 & 9 \\
\hline Mucous challenge test $(n=45)$ & $11(\mathrm{TSS}=13)$ & $4(\mathrm{TSS}=10)$ \\
\hline Nasal challenge test $(n=26)$ & $24(\mathrm{TSS}=13)$ & $5(\mathrm{TSS}=8)$ \\
\hline Conjuctival challenge test $(n=46)$ & $42(\mathrm{TSS}=71)$ & $27(\mathrm{TSS}=50)$ \\
\hline
\end{tabular}

TSS - total symptoms score.

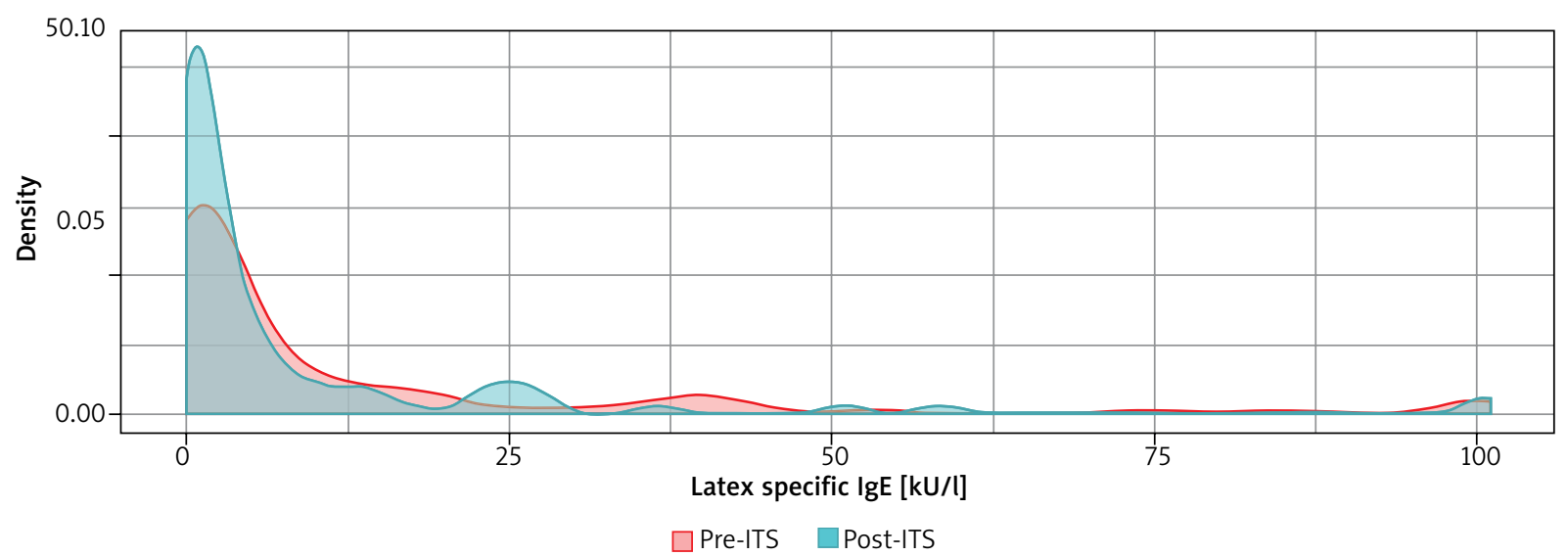

Figure 2. Latex specific IgE before and after sublingual immunotherapy (ITS)

At the beginning of the study, a cutaneous provocation test was carried out in 72 out of 76 patients because the remaining 4 patients presented dermatitis of their hands. During the test, 57 subjects developed adverse reactions (rhinoconjunctivitis, cutaneous symptoms or both); of these subjects 48 became negative at the end of the trial $(p<0.01)$. Anyway, regarding the other $9 / 57$ patients who remained positive at the cutaneous test, no changes were observed in the total symptoms score before and after the SLIT.

The other challenges (mucous-oral, conjunctival and nasal) have been performed in a limited number of patients because not all the subjects gave their consent to undergo the procedures.

The mucous challenge test, performed in 45 out of 76 subjects, scored positive in 11 of them; after the immunotherapy 4 patients still reacted but there was a slight decrease in the total symptoms score that cut down from 13 to $10(p=0.003)$.

Before the treatment, the nasal provocation test was performed in 26 out of 76 patients and 24 subjects were positive: after 10-45 min, they presented rhinoconjunctivitis or cutaneous symptoms. After 3 years of immunotherapy only 5 out of 24 patients were still positive but we observed a slight reduction in total symptoms score from a value of 13 to 8 but this result, although interesting, was not statistically significant.

Concerning 46 out of 76 patients who performed the conjunctival test, we observed a clear negativity in 15 out of 42 subjects who had an adverse reaction during this test before the treatment $(p<0.05)$. In patients who were still positive to this challenge after the desensitization there was a decrease in the total symptoms score from 71 to 50 .

During the trial, 35/76 patients came into contact accidentally with latex: 25 of them did not present an adverse reaction, while 10/35 developed an allergic reaction which consisted of mild cutaneous symptoms in all cases and one patient had a systemic reaction.

\section{Discussion}

The main therapeutic approach in latex sensitized subjects is to avoid latex exposure. In patients that have difficulties in applying adequate avoidance measures, a sublingual immunotherapy is recommended.

Our team has already widely published on this matter. 
In a publication of 2002 [4], we evaluated the efficacy of latex immunotherapy; we randomly assigned 12 patients as controls and 12 patients undergoing treatment according to the rush protocol. All patients performed cutaneous, mucosal, sublingual and conjunctival challenges, evaluating a symptoms score (baseline and after 3 months). In the active group, we observed a significant reduction in latex specific IgE values in 7 patients and a negative outcome in cutaneous, mucosal and sublingual challenges, while the conjunctival one showed only a significant symptoms score reduction. In conclusion, this study showed how the latex immunotherapy in 4-day rush protocol is effective in patients with a latex allergy.

In a further experience [15], our team compared 2 different induction rush protocols ( 2 and 3 days) in terms of efficacy, safety and tolerability in reaching the maintenance dose. The patients were randomized: 10 in group 1 ( 2 days) and 12 in group 2 (3 days). The skin prick test became negative in 16 patients ( 8 in both group 1 and 2 ) and the remaining patients showed a significant symptoms score reduction.

Other authors support the safety and efficacy of immunotherapy (SLIT) for latex.

Buyukozturk et al. [8] demonstrated a clear efficacy of desensitisation after 12 months' treatment showing a reduction of symptoms and scores at the cutaneous provocation tests performed in allergic patients (all health professionals who were unable to prevent the clinical manifestations with the prevention procedures only). The same results were obtained by Bernardini et al. [16].

Lasa Luaces [17] showed both the effectiveness (increased triggering dose during the conjunctival provocation test) and immunological changes after 6 and 12 months of treatment. In his work the IgG4 appeared to increase between 6 and 12 months of therapy, while the basophil activation test with latex showed a reduction of reactivity after 6 months. The IgE instead did not show statically significant variations.

Cisterò Bahima et al. [18] sought to evaluate the tolerability of SLIT latex and its effects on skin reactivity. Twenty-six allergic patients (history of skin and respiratory symptoms after latex contact) underwent SLIT (4 days of induction with 9 weeks of maintenance). The cutaneous challenge, the rubbing test and the SPT were carried out before, during and at the end of SLIT. The cutaneous provocation test was greatly improved both after 5 days and 10 weeks of treatment, while the rubbing test only after 10 weeks. No changes in the SPT were detected.

On the other hand, in the literature there are also cases that show lack of efficacy of the treatment: Morfin Maciel et al. [19] described the case of a boy who presented anaphylaxis after inspection in the airport with latex gloves despite latex immunotherapy. Gastaminza et al. [20] showed that there is no significant statistical difference between the active and placebo group either in vivo or in vitro. Moreover this group highlighted a reduction in the percentage of basophil activation both in the active group after 2 years of therapy and in the placebo group after 1 year of treatment.

The aim of our study was to update the data of the literature about the management of the latex allergy in those patients in whom the avoidance measures were not feasible or effective. The patients underwent latex SLIT for at least 3 years during which we checked the clinical and immunological changes comparing the outcomes of the provocation test, skin prick test and in vitro test.

Although in our study there was no control group, it must be highlighted that we enrolled a larger group of patients than the studies described above.

In contrast with the observations of Lasa Luaces, in this work we observed a decrease in latex specific IgE as previously stated $[4,8]$ and no statistically significant changes of latex IgG4 values were found. On the other hand, we did not confirm the data of Cisterò Bahima, detecting a significant reduction in the mean diameter of the wheal area at SPT.

According to Cisterò Bahima, Buyukoturk, Bernardini and Lasa Luaces, a reduction of symptoms and scores at the provocation tests were remarked.

\section{Conclusions}

Although the primary prevention (and thus the complete elimination of latex contact) still remains the gold standard treatment for patients suffering from the latex allergy, SLIT can be offered with efficacy in addition to symptomatic treatment to selected patients.

For these reasons, further investigations in this field are needed, especially in order to identify tolerability, safety, long-term effectiveness and maintenance dosage, as the literature shows they are still widely variable.

\section{Conflict of interest}

The authors declare no conflict of interest.

\section{References}

1. Niggemann B. IgE-mediated latex allergy - an exciting and instructive piece of allergy history. Pediatr Allergy Immunol 2010; 21: 997-1001.

2. Rolland JM, O'Hehir RE. Latex allergy: a model for therapy. Clin Exp Allergy 2008; 38: 898-912.

3. Chełmińska M, Specjalski K, Różyło A, et al. Differentiating of cross-reactions in patients with latex allergy with the use of ISAC test. Adv Dermatol Allergol 2016; 33: 120-7.

4. Allmers H, Schmengler J, John SM. Decreasing incidence of occupational urticaria caused by natural rubber latex allergy in German health care workers. J Allergy Immunol 2004; 114: 347-51. 
5. Patriarca G, Nucera E, Pollastrini E, et al. Sublingual desensitization: a new approach to latex allergy problem. Anesth Analg 2002; 95: 956-60.

6. Cistero Bahima A, Sastre J, Enrique E, et al. Tolerance and effects on skin reactivity to latex of sublingual rush immunotherapy with a latex extract. J Investig Allergol Clin Immunol 2004; 14: 17-25.

7. Nettis E, Colanardi MC, Soccio Al, et al. Double-blind, placebo-controlled study of sublingual immunotherapy in patients with latex-induced urticaria: a 12-month study. $\mathrm{Br}$ J Dermatol 2007; 156: 674-81.

8. Buyukozturk S, Gelincik A, Özseker F, et al. Latex sublingual immunotherapy: can its safety be predicted. Ann Allergy Asthma Immunol 2010; 104: 339-42.

9. Durham SR, Emminger W, Kapp A, et al. Long-term clinical efficacy in grass pollen-induced rhinoconjunctivitis after treatment with SQ-standardized grass allergy immunotherapy tablet. J Allergy Clin Immunol 2010; 125: 131-8.

10. Pajno GB, Morabito L, Barberio G, Parmiani S. Clinical and immunologic effects of long-term sublingual immunotherapy in asthmatic children sensitized to mites: a double-blind, placebo-controlled study. Allergy 2000; 55: 842-9.

11. Patriarca G, Schiavino D, Pecora V, et al. Food allergy and food intolerance: diagnosis and treatment. Intern Emerg Med 2009; 4: 11-24.

12. Malling HJ, Weeke B. Immunotherapy. Position paper of EAACl. Allergy 1993; 48 (Suppl. 14): 9-35.

13. Dreborg S, Frew A. Allergen standardization and skin tests. Allergy 1993; 48: 49-75.

14. Nucera E, Pollastrini E, Sabato V, et al. Challenge tests in the diagnosis of latex allergy. Int J Immunopathol Pharmacol 2010; 23: 543-52.

15. Nucera E, Schiavino D, Sabato V, et al. Sublingual immunotherapy for latex allergy: tolerability and safety profile of rush build-up phase. Curr Med Res Opin 2008; 24: 1147-54.

16. Bernardini R, Pecora S, Milani M, Burastero SE. Natural rubber latex allergy in children: clinical and immunological effects of 3-years sublingual immunotherapy. Eur Ann Allergy Clin Immunol 2008; 40: 142-7.

17. Lasa Luaces EM, Tabar Purroy Al, García Figueroa BE, et al. Component-resolved immunologic modifications, efficacy, and tolerance of latex sublingual immunotherapy in children. Ann Allergy Asthma Immunol 2012; 108: 367-72.

18. Cisterò Bahima A, Sastre J, Enrique E, et al. Tolerance and effects on skin reactivity to latex of sublingual rush immunotherapy with a latex extract. J Investig Allergol Clin Immunol 2004; 14: 17-25.

19. Morfin Maciel BM, Castillo Morfin BM. Failure of sublingual immunotherapy to treat latex allergy. A report of a case. Rev Alerg Mex 2008; 55: 76-81.

20. Gastaminza G, Algorta J, Uriel O, et al. Randomized, doubleblind, placebo-controled clinical trial of sublingual immunotherapy in natural rubber latex allergic patients. Trials 2011; 12: 191. 